\title{
ORIGINAL
}

\section{EVALUACIÓN DE LA VIGILANCIA EN SALUD EN ALGUNAS UNIDADES DE ATENCIÓN PRIMARIA EN CUBA}

Ricardo Batista Moliner (1), Edilberto González Ochoa (2) y Pablo Feal Cañizares (1)

(1) Unidad de Análisis y tendencias en salud. MINSAP, Cuba

(2) Instituto de Medicina Tropical Pedro Kouri, Cuba

\section{RESUMEN}

Fundamento. Se evaluó la organización y funcionamiento de la vigilancia epidemiológica en varias unidades de la Atención Primaria de Salud en Cuba, mediante un estudio para conocer la efectividad de la vigilancia y contribuir a perfeccionar los mecanismos de la misma y a través de ella mejorar las condiciones de salud de la población.

Método. La evaluación se aplicó en ocho Policlínicos de Ciudad de La Habana durante los años 1998 y 1999. Mediante la aplicación de una metodología integral se evaluaron los tres componentes del sistema de vigilancia a esas unidades: la estructura, el proceso y sus resultados. Para ello se utilizó un cuestionario que cuantificó algunos atributos del sistema (sensibilidad, oportunidad, representatividad, utilidad) y una entrevista semiestructurada a médicos, epidemiólogos y a otros participantes en la vigilancia, así como a los usuarios del sistema.

Resultados. La evaluación de la estructura demostró que la estructura de vigilancia es adecuada. Al evaluar el proceso se comprobó que la sensibilidad es baja en algunos policlínicos y que en varios de ellos existen problemas en la oportunidad y flexibilidad del sistema. La evaluación de los resultados de la vigilancia demostró que éstos son satisfactorios en su eficacia para detectar eventos agudos, así como en la percepción de los usuarios sobre la utilidad de esos resultados para resolver los problemas detectados.

Conclusiones. El desempeño de la vigilancia en los policlínicos evaluados refleja que existe una adecuada organización de la vigilancia, aunque existen algunas insuficiencias en el cumplimiento de algunos atributos de la vigilancia. Esto, sin embargo, no afecta el resultado del sistema en su conjunto, que es capaz de detectar cualquier situación epidemiológica relevante y proponer medidas adecuadas de control.

Palabras clave. Estudios de evaluación. Vigilancia en salud. Atención Primaria.

Correspondencia:

Ricardo Batista Moliner

Bellavista 516, Apto 14 e/ Colón y 39

Nuevo Vedado, Plaza de la Revolución

Ciudad de la Habana

Cuba

\section{ABSTRACT}

\section{Assessment of Public Health Monitoring at some Primary Care Units in Cuba}

Background: The organization and operation of the epidemiological monitoring carried out at several Primary Care facilities in Cuba was assessed by means of a study conducted for the purpose of ascertaining the effectiveness of the monitoring in question and of contributing to further improve the mechanisms thereof and thus improve the health conditions of the population. This assessment was implemented at eight Polyclinics in the city of Havana throughout the 1998-1999 period.

Method: By means of the implementation of an integral methodology, an assessment was made of the three factors involved in the monitoring system at the facilities in question, that is, the organization, the process and the results thereof. For this purpose, a survey which quantified some aspects of the system (sensitivity, opportunity, representativeness, usefulness) and a semi-structured survey of epidemiologists and other physicians involved in the monitoring in question in addition to the users of the system was used.

Results. The assessment of the organization revealed the monitoring organization to be adequate. In the assessment of the process, sensitivity was found to be low at some polyclinics, problems existing at many of them with regard to the opportunity and flexibility of the system. The assessment of the results of the monitoring revealed them to be satisfactory as regards their effectiveness for detecting acute events, as well as in the perception of the users with regard to the usefulness of these results for solving the problems pinpointed.

Conclusions: The monitoring done at the polyclinics assessed is indicative of there being an adequate organization of the monitoring, although some shortcomings were found to exist with regard to the fulfillment of some aspects of the monitoring. This however has no bearing on the results for the system as a whole, which is capable of detecting any epidemiological situation of importance and of suggesting appropriate measures for controlling the situation. Care.

Key words: Assessment. Health Monitoring. Primary 


\section{INTRODUCCIÓN}

Los avances alcanzados por la Salud Pública a nivel internacional han traído consigo un desarrollo de la vigilancia epidemiológica, introduciendo nuevos enfoques y dirigiendo su trabajo hacia una vigilancia más amplia e integral que se identifica actualmente como vigilancia en salud pública. La misma se ha definido como un proceso esencial para asegurar la identificación de los eventos de salud más relevantes y desarrollar las acciones necesarias para su control $^{1}$.

Sobre la base de estas nuevas concepciones y la experiencia acumulada hasta el momento, en Cuba se ha redimensionado y desarrollado un modelo de vigilancia en salud que responda a las necesidades del sistema sanitario, de acuerdo con los cambios del patrón epidemiológico y las transformaciones operadas en las condiciones de salud de la población. La vigilancia en salud se ha ido desarrollando en el país y sus resultados tienen un impacto decisivo en la mejoría de la gestión y de los indicadores de salud a todos los niveles. Estos resultados dependen en una amplia medida del desempeño de la vigilancia en la Atención Primaria, instancia en la cual tienen lugar las principales incidencias relacionadas con la salud, ya sean de riesgo o daños; reconocida como el primer nivel de contacto de los individuos, la familia y la comunidad con el sistema de salud y por tanto el primer escalón del proceso de atención a la salud de la población ${ }^{2}$.

La Atención Primaria en Cuba constituye la base principal del sistema de salud y se articula asistencialmente al nivel secundario y terciario a través de los Policlínicos, que representan la unidad que concentra la información y mantiene una estrecha vinculación con los consultorios médicos directamente en las comunidades. Administrativamente estas unidades de salud se subordinan a la instancia municipal, hacia donde se transmite la información recolectada de dichas unidades.
Esta sólida red sanitaria facilita la ejecución de actividades asistenciales, de control, promoción y prevención de salud en todo el país. La vigilancia en este nivel de atención es decisiva entonces para el éxito de las acciones e intervenciones sobre los problemas identificados; por tanto la evaluación de su funcionamiento y de sus resultados resultan de vital importancia en el proceso de gestión de la Salud Pública, en busca de mayor efectividad y eficiencia.

En etapas anteriores al proceso de reorganización del sistema de salud se hicieron varios intentos de evaluación de algunos atributos de la vigilancia en determinados problemas de salud en el país, la mayoría de ellos no publicados. Posteriormente se realizaron otros, aunque de limitado alcance en su aplicación.

La evaluación como proceso de búsqueda de elementos que permitan emitir un juicio de valor sobre una actividad u objeto, requiere de un riguroso proceso de recogida de la información, necesaria para conformar un criterio lo más cercano posible a lo que se ha logrado o lo que realmente es con determinada actividad, con relación a lo que se debía lograr o ser. La evaluación, por tanto, es un procedimiento complejo, el cual ha sido abordado y desarrollado por numerosos autores y se concibe cada vez más como un proceso de análisis e investigación dirigido a estimar el valor y la contribución de cada tecnología sanitaria a la mejora de la salud individual y colectiva, teniendo en cuenta su impacto económico y social ${ }^{3,4}$. Ejemplos de estudios realizados para evaluar algunos atributos de la vigilancia son el estudio de la sensibilidad y oportunidad de la vigilancia intensiva de hepatitis en Minnesota ${ }^{5}$, el de la vigilancia de la mortalidad por influenza en Estados Unidos 6 ; el de la sensibilidad y oportunidad de la vigilancia y detección de brotes de enfermedades infecciosas ${ }^{7}$; el de la sensibilidad y valor predictivo positivo de la certificación de la muerte ${ }^{8}$, y el de la vigilancia de la brucelosis en el País Vasco, España ${ }^{9}$. En la practica los atributos que ma- 
yor atención han recibido son la sensibilidad y el valor predictivo positivo ${ }^{10-12}$.

Los criterios para la evaluación de la vigilancia han sido expuestos por especialistas de distintas instituciones especializadas en el tema, los cuales señalan el valor de tener en cuenta: elementos de la organización del sistema, la importancia del problema a vigilar, así como la evaluación de varios atributos como la sensibilidad, oportunidad, flexibilidad, valor predictivo positivo o representatividad, entre otros ${ }^{13}$. En Cuba los estudios de evaluación de la vigilancia son escasos y se han dirigido a problemas específicos.

Sobre la base de estos antecedentes, el objetivo de nuestro trabajo fue evaluar el funcionamiento del sistema de vigilancia a nivel de la Atención Primaria de Salud en varios Policlínicos de la provincia Ciudad de La Habana y tener una apreciación de su desempeño, identificando algunos de los problemas que afectan la vigilancia en centros de salud primarios en este territorio.

\section{SUJETOS Y MÉTODO}

Se realizó una evaluación de la vigilancia en ocho Policlínicos de Ciudad de La Habana, durante los años 1998 y 1999. Para realizar el estudio se aplicó un cuestionario con una serie de preguntas que responden a los diferentes componentes y atributos del sistema de vigilancia. El estudio se realizó en el territorio centro-oeste de la Ciudad de la Habana, constituido por los municipios Plaza, Cerro, Centro Habana, Playa, Lisa y Marianao. Esto se determinó así debido a que en esta zona de la provincia se desarrolla un trabajo de perfeccionamiento de la vigilancia en la APS que incluye la evaluación de la vigilancia en dichos centros de salud. Además, estas unidades de salud constituyen áreas de practica de campo de epidemiología del Instituto Pedro Kouri. En total en este territorio existen 24 Policlínicos.
Atendiendo a la opinión de un grupo de expertos de toda la provincia, integrado por epidemiólogos y técnicos de higiene, se decidió realizar el estudio en cuatro municipios de esta región: Plaza, Cerro, Marianao y Playa, pues no se consideró necesario aplicar un muestreo probabilístico de estos municipios. Tómese en cuenta que el proceso de vigilancia en el país sigue orientaciones uniformes para esta actividad y las características de estructura y funcionamiento son muy homogéneas (UATS. La vigilancia en Salud en Cuba, Area de Higiene y Epidemiología, Ministerio de Salud Publica, 1996; Carpeta Metodológica del Ministerio de Salud Publica, 1997).

Siguiendo un criterio de factibilidad y la existencia de 4 equipos de trabajo se escogió al azar un Policlínico en cada municipio donde se realizaría el estudio. El mismo procedimiento se repitió de forma similar en el segundo año, es decir se seleccionaron 4 unidades en 1998 y 4 en 1999. En la evaluación participaron alumnos de la Maestría de Epidemiología del curso correspondiente a los dos períodos académicos en los que se realizaron las evaluaciones (1997/98 y 1998/99). Estos alumnos fueron adiestrados en la aplicación del procedimiento de evaluación.

De acuerdo con el cuestionario, la respuesta a cada pregunta se califica con una puntuación de 0 y 1 , para las preguntas sobre estructura, atributos cualitativos (simplicidad, flexibilidad, aceptabilidad, integralidad, capacidad de autorrespuesta) y sobre resultados, mientras que en las preguntas sobre los atributos cuantitativos los valores de respuesta se califican de 1 a 4 (Anexo $1)^{14}$. Esta metodología fue previamente validada, mediante la aplicación del cuestionario en cuatro Policlínicos de Ciudad de la Habana, en el año $1996^{14}$.

Para evaluar el sistema el procedimiento lo divide en sus tres componentes: estructura, proceso y resultados, según el enfoque recomendado por Donabedian ${ }^{3}$. En la es- 
tructura se tienen en cuenta dos elementos, el sujeto referido a la organización de los recursos materiales y humanos (médicos, enfermeras, técnicos) que ejecutan las actividades de vigilancia y el objeto concerniente a la población y la comunidad sobre la cual se desarrolla la vigilancia.

El proceso corresponde con el análisis del cumplimiento de los atributos básicos de un sistema de vigilancia para que se considere efectivo: simplicidad, aceptabilidad, flexibilidad, sensibilidad, valor predictivo positivo (VPP), oportunidad, representatividad, según se han definido para estos sistemas ${ }^{13}$. En este caso se incluyeron dos parámetros adicionales: integralidad y capacidad de autorrespuesta. El primero se refiere a la capacidad para analizar de forma integral y completa los eventos vigilados, desde el punto de vista ambiental, social y en el ámbito individual, así como de grupos específicos o de la comunidad; mientras que el segundo se refiere a la capacidad del sistema de proponer y estimular acciones para el control de los eventos detectados ${ }^{15}$. Para evaluar la sensibilidad y el VPP se utilizó el sistema de reporte Alerta-Acción como procedimiento para la vigilancia y se contrastó con el sistema de confirmación y registro definitivo de Enfermedades de Declaración Obligatoria (EDO) como modelo de referencia, según se muestra:

\begin{tabular}{c|c|c|c|}
\multicolumn{1}{c|}{} & \multicolumn{2}{c}{ EDO } \\
\cline { 3 - 4 } \multicolumn{1}{c|}{} & + & - \\
\hline \multirow{3}{*}{ Alerta- } & + & $\mathrm{a}$ & $\mathrm{b}$ \\
\cline { 2 - 4 } Acción & - & $\mathrm{c}$ & $\mathrm{d}$ \\
\cline { 2 - 4 } & & &
\end{tabular}

Para evaluar estos dos atributos se realizó el análisis de tres enfermedades: Tuberculosis, Blenorragia y Leptospirosis, por ser estas entidades de las que se mantienen sujetas a un estricto control y seguimiento en todo el país, cuyas definiciones se recogen en dichos programas ${ }^{17-19}$. Los datos para validar el reporte de las unidades se obtuvieron de los registros estadísticos a nivel municipal.
El análisis se realizó para cada enfermedad en todas las unidades evaluadas. El valor global de sensibilidad y VPP, en el Policlínico se estimó como el promedio del índice calculado para cada enfermedad individual. Para obtener información adicional sobre los atributos cualitativos se aplicaron encuestas a participantes (Anexo 2).

Con respecto a los resultados se dirigió el análisis a determinar si el sistema cumple con los propósitos para los cuales está concebido, es decir su capacidad para detectar brotes, epidemias, casos o eventos agudos relevantes, y si además resulta útil a los principales usuarios.

Para ejecutar el proceso de evaluación se realizaron entrevistas a los directivos de los Policlínicos (directores y vicedirectores, jefes de departamento); además se revisaron documentos, actas de reuniones técnicas, reportes, informes elaborados como resultados de los análisis realizados, incluido el análisis de la situación sanitaria del Area de salud. Los datos necesarios para el análisis de los atributos cuantitativos, se obtuvieron de los registros estadísticos del Policlínico. También se realizó una observación del proceso de análisis, participando en alguna de las reuniones donde se realizaron los análisis de los problemas bajo vigilancia. En estas entrevistas y actividades se indagaron y comprobaron también los aspectos necesarios para responder a las preguntas del cuestionario de evaluación, en particular para calificar aquellas relacionadas con los atributos cualitativos.

Otros participantes, es decir personal que ejecuta las actividades de vigilancia (médicos, enfermeras y técnicos) y usuarios del sistema de vigilancia, o sea los que reciben los resultados del mismo; se encuestaron mediante la aplicación de cuestionarios estructurados autoadministrados (Anexo 2). Como participantes se encuestaron representantes de cada instancia dentro de la estructura del Area de Salud. Se escogieron aleatoriamente 12 médicos de familia que 
laboran en consultorios, un jefe de Grupo Básico de Trabajo (GBT), así como el jefe técnico de los Departamentos del Policlínico (Laboratorio, Estadísticas e Higiene) y un representante del Consejo de Dirección del Policlínico; por ser estos los principales involucrados en el proceso de vigilancia y con los cuales se exploraron aspectos como la simplicidad, aceptabilidad y la retroalimentación; sirviendo para calificar las preguntas correspondientes del cuestionario de evaluación.

En el caso de los usuarios se escogió un representante de cada institución o entidad extrasectorial a los cuales se dirigen las comunicaciones y los resultados de la vigilancia a ese nivel, por ser éstos los que deben recibir la información correspondiente de acuerdo con la concepción y organización del sistema en el país. Estos incluyen el gobierno local, organizaciones de políticas, sociales y de masas y entidades administrativas del territorio. Para ello se utilizó también un cuestionario estructurado auto administrado (Anexo 3). Fueron encuestados entonces los Presidentes de Consejo Popular y Delegados de circunscripción, coordinadores o responsables de Servicios Comunales y líderes formales de la comunidad. En este caso el propósito fue comprobar si recibían la información de los resultados de la vigilancia y la utilidad que les reportaba dicha información.

Para definir los resultados finales se determinó la puntuación de cada componente y atributo evaluado, entre los obtenidos por cada una de las unidades. Esta calificación se determinó en base a los resultados obtenidos en un proceso de pilotaje realizado durante la validación del instrumento de evaluación y aplicación de la metodología; resultando en el esquema de calificación siguiente:

Estructura. Puntuación máxima: 19 puntos.

- 0 a $10=$ Estructura mal definida.

- 11 a $13=$ Estructura con imprecisiones.

- 14 a $19=$ Estructura adecuadamente definida.

Sujeto (13 puntos): indefinido (0 a 6)

poco definido (7 a 9 )

bien definido (10 a 13)

Objeto (6 puntos): indefinido (0 a 2)

poco definido (3 a 4 )

bien definido ( 5 a 6 )

Proceso. Puntuación máxima: 97 puntos.

- 0 a 32 puntos $=$ Funcionamiento deficiente.

- 33 a 65 puntos $=$ Funcionamiento regular.

- 66 a 97 puntos $=$ Buen funcionamiento.

Resultados: Puntuación máxima: 13 puntos.

-0 a 5 puntos $=$ No útil.

-6 a 9 puntos $=$ Aceptable.

-10 a 11 puntos $=$ Util

- 12 o más puntos = Muy útil. 


\begin{tabular}{|c|c|c|c|c|}
\hline Atributo del proceso & Total puntos & Mala & Regular & Buena \\
\hline Simplicidad & 12 & 0 a 6 & 7 a 10 & 11 a 12 \\
\hline Aceptabilidad & 3 & 0 a 1 & 2 & 3 \\
\hline Flexibilidad & 3 & 0 a 1 & 2 & 3 \\
\hline Integralidad & 3 & 0 a 1 & 2 & 3 \\
\hline Sensibilidad & 8 & 0 a 2 & 3 a 5 & 6 a 8 \\
\hline VPP & 4 & 0 a 2 & 3 & 4 \\
\hline Oportunidad & 16 & 0 a 8 & 9 a 13 & 14 a 16 \\
\hline Capacidad de Autorrespuesta & 20 & 0 a 10 & 11 a 17 & 18 a 20 \\
\hline Representatividad & 28 & 0 a 14 & 15 a 22 & 23 a 28 \\
\hline
\end{tabular}

\section{RESULTADOS}

Al evaluar la estructura se comprobó una organización adecuadamente definida, de acuerdo con la puntuación obtenida. Ambos componentes considerados, sujeto y objeto de vigilancia, estaban bien definidos, según se aprecia en la tabla 1. En todas las unidades evaluadas puede decirse que tanto la infraestructura de salud (recursos humanos y materiales) encargada de desarrollar las actividades de vigilancia, como la población y la comunidad sobre la cual se aplica la misma mantenían una buena definición, de acuerdo con las necesidades del sistema y aseguraban su funcionamiento.

Tabla 1

Evaluación de la estructura. Ciudad de la Habana, 1998-99

\begin{tabular}{|lrl|}
\hline & $\begin{array}{c}\text { Puntuación } \\
\text { media }\end{array}$ & \multicolumn{1}{c|}{ Calificación } \\
\hline Sujeto & 10,33 & Bien definido \\
Objeto & 5,78 & Bien definido \\
Estructura & 16,11 & Adecuadamente definida \\
\hline
\end{tabular}

Para evaluar el proceso se consideró el cumplimiento de los atributos básicos de la vigilancia, es decir la simplicidad, aceptabilidad, flexibilidad, oportunidad, sensibilidad, valor predictivo positivo, así como la integralidad y capacidad de autorrespuesta.

De acuerdo con los resultados obtenidos, que se presentan en la tabla 2, cinco de los nueve atributos, se califican de regular, tres de ellos tienen un mal desenvolvimiento en algunas unidades evaluadas y solo la representatividad se expresa adecuadamente en el desarrollo del proceso. De forma general en la evaluación de estos atributos, las mayores dificultades se presentan en el caso de la sensibilidad y el VPP, pues en algunos casos al revisar en detalles los registros primarios de notificación, se encontraron valores muy diferentes entre las Areas de Salud evaluadas. Para evaluar este atributo se utilizaron como problemas la Tuberculosis, la Blenorragia y la Leptospirosis, lo que aportó valores de sensibilidad bajos en la mayoría de los casos, entre 45 y $60 \%$, lo que evidencia que algunas de las Areas de Salud presentan dificultades para identificar enfermos en esa instancia y son diagnosticados en el nivel superior, es decir hospitales o institutos.

En el caso del VPP los resultados también indican una puntuación muy baja, lo que puede atribuirse al hecho de ser entidades de baja frecuencia, en las que se reportan dos o tres casos en cada Policlínico al año para la Tuberculosis y Leptospirosis; y de menos de 20 para la Blenorragia. Además se registra un número elevado de sospechosos, dando cumplimiento a las estrategias de pesquisa de los programas de prevención y control de dichas entidades en el país.

Otros atributos mostraron resultados variables en las diferentes Areas de salud estudiadas. Con respecto a la simplicidad algunos Policlínicos señalan exceso de modelos a confeccionar y complejidad de los mismos para notificar los problemas. La flexibilidad se calificó como deficiente solo en un Area de salud donde el sistema no se adecuaba satisfac- 
Tabla 2

Evaluación del proceso. Ciudad de la Habana, 1998-99

\begin{tabular}{|c|c|c|c|}
\hline Atributo & Puntuación a obtener & Puntuación media & Calificación \\
\hline Simplicidad & 12 & 7,33 & Regular \\
\hline Aceptabilidad & 3 & 2,22 & Regular \\
\hline Flexibilidad & 3 & 1,78 & Mal \\
\hline Integralidad & 3 & 2,56 & Regular \\
\hline Sensibilidad & 8 & 3,86 & Regular \\
\hline VPP & 4 & 1,71 & Mal \\
\hline Oportunidad & 16 & 10,22 & Mal \\
\hline Capacidad de Autorrespuesta & 20 & 13,89 & Regular \\
\hline Representatividad & 28 & 24,5 & Bien \\
\hline Proceso & 97 & 64,11 & Funcionamiento Regular \\
\hline
\end{tabular}

toriamente para satisfacer la necesidad de dar seguimiento a problemas relevantes. Este problema se analizó para el caso de la Conjuntivitis hemorrágica que presenta eventualmente situaciones epidémicas en el país.

La capacidad para identificar rápidamente los eventos y actuar oportunamente en general se determinó como satisfactoria, aunque en muchos de ellos no existen los medios automatizados que agilizan el proceso de recogida y procesamiento de los datos. Finalmente la capacidad de autorrespuesta del sistema, en función de estimular las respuestas convenientes ante los problemas detectados, a partir de las recomendaciones del sistema de vigilancia, se identificó con dificultades en dos Areas de Salud, al contar con poca participación intersectorial; en el resto fue efectiva. En resumen el proceso fue evaluado de funcionamiento regular en 5 Policlínicos y en tres la calificación indicó un buen funcionamiento.

De acuerdo con lo analizado para conocer los resultados, se determinó que el sistema alcanza los objetivos para los que ha sido concebido, siendo capaz de identificar casos y situaciones agudas de importancia epidemiológica. Aunque la puntuación más baja se registró en dos Policlínicos; el índice medio fue de 9 , considerándose adecuado este aspecto del sistema.

En las entrevistas a los participantes se pudo comprobar que, en general, los mis- mos plantean que reciben periódicamente información del sistema de vigilancia, aunque en ocasiones es de forma irregular o tardíamente. Entre los elementos que señalan como responsable de esas irregularidades están la falta de medios materiales para hacer llegar mas rápidamente los datos a todos los participantes en el sistema (equipos de computación y reproducción, papel, etc.) y por otro lado el exceso de actividades administrativas que les impide actualizarse en relación con los principales problemas de salud del área o territorios aledaños.

Mediante las encuestas a usuarios se comprobó que más del $80 \%$ de ellos, recibe sistemáticamente información de la vigilancia a través del Consejo mensual de Salud. Algunos de los entrevistados fueron capaces de expresar problemas y situaciones de salud identificadas por la vigilancia, especialmente se mostraron satisfechos los representantes de la comunidad (autoridades de gobierno y administrativas). De igual forma la mayor parte de las entrevistas indicaron que la información recibida les resultó útil para realizar su trabajo en función de los problemas detectados y para tomar decisiones sobre las acciones y medidas necesarias que faciliten la mejoría de las condiciones de salud de la comunidad que atienden.

Fue muy ilustrativo de la correspondencia entre el proceso de vigilancia y sus resultados, que en todos los Policlínicos donde se 
determinó un buen funcionamiento del sistema, fue calificado de muy útil, de acuerdo con los resultados y los criterios de los usuarios y participantes en el mismo

\section{DISCUSIÓN}

De acuerdo con los resultados obtenidos puede decirse que la organización del sistema de vigilancia en los Policlínicos evaluados fue satisfactoria, si se tiene en cuenta que disponen del personal necesario, adecuadamente preparado y los recursos mínimos para su buen desempeño; aun cuando los medios materiales no son los óptimos para desarrollar esta labor, especialmente por la escasez de equipos automatizados que agilizan las tareas y facilitan muchos procedimientos, las cuales se realizan manualmente en la mayoría de esos centros.

El sistema de vigilancia en estas unidades se adapta a la estructura general del sistema de salud, respondiendo a las necesidades de la comunidad en la que se desarrolla, de acuerdo a su magnitud y trascendencia. En otros estudios para evaluar el sistema de vigilancia de la sífilis congénita en nuestro país, se constató que la estructura para desarrollar estas actividades de vigilancia es satisfactoria, tanto en términos de cobertura como de preparación del personal que la ejecuta $^{20,21}$. En las áreas evaluadas se comprobó la presencia de más del $95 \%$ del personal necesario para realizar esta actividad, ya fueran profesionales o técnicos. Sin embargo, en otro estudio realizado en Ciudad de La Habana se determinó que la cobertura y preparación del personal para realizar la vigilancia era deficiente ${ }^{22}$.

Los principales problemas identificados están relacionados con su funcionamiento y básicamente con algunos atributos del mismo, lo que afecta a su desempeño de forma óptima. Tal es el caso de la flexibilidad y la oportunidad, aunque éstas no parecen afectar al resultado final del sistema. La sensibilidad y el VPP son dos atributos de gran importancia en la efectividad del sistema, presentaron resultados desfavorables, lo que nos explicamos por el hecho de utilizar para su análisis enfermedades de baja incidencia a este nivel y que además son objeto de programas que promueven la búsqueda activa de casos para reducir su transmisión en la comunidad, lo que recarga el sistema con un alto numero de sospechosos que luego son descartados. Ante esta limitación, se ha sugerido seleccionar otros problemas de salud de mayor frecuencia o utilizar para el análisis otro procedimiento comparativo de lo detectado por la vigilancia, lo que permitiría evaluar mas objetivamente estos atributos, como es el método de captura-recaptura, que puede ser valido para el análisis de otros problemas. En estudios realizados para la evaluar la vigilancia de la sífilis congénita se obtuvieron resultados muy favorables con valores por encima del $98 \%$, utilizando valores esperados para la entidad evaluada ${ }^{16,20}$.

Con relación a la oportunidad, en el mencionado estudio de evaluación de la vigilancia de la sífilis congénita en la capital, se detectó un retraso en el tiempo que transcurre entre la toma de la muestra y la notificación del resultado.

Finalmente, a pesar de estos elementos planteados sobre el comportamiento de algunos atributos del sistema que limitan que el mismo tenga un funcionamiento óptimo, particularmente de la sensibilidad y VPP, no se afectan los resultados finales de la vigilancia en estas unidades y permiten identificar eventos de salud relevantes y la aplicación de acciones oportunas, lo que, a juicio de los autores, tiene que ver con la alta prioridad y atención que tiene la detección rápida y control de cualquier situación de salud que ponga en riesgo el bienestar de toda la comunidad. Por esa razón se considera útil por los usuarios del mismo.

La estructura del sistema de vigilancia en las unidades estudiadas está adecuadamente definida y organizada para satisfacer las necesidades del mismo. El funcionamiento y el cumplimiento de los principales atributos del sistema, aunque para algunos Policlíni- 
cos se reconocen bajos indicadores, alcanza los objetivos en varios de los centros de salud evaluados y se consideran útiles por los que reciben sus resultados. Los principales problemas se comprobaron en la oportunidad, la sensibilidad y el VPP.

\section{AGRADECIMIENTOS}

Al estimado amigo Dr. Dionisio Herrera por su inapreciable ayuda en la revisión del manuscrito y sus acertadas sugerencias en la redacción final de informe.

Anexo 1

Cuestionario utilizado para la evaluación del sistema de vigilancia

\begin{tabular}{|c|c|c|}
\hline Evaluación de la estructura & \multicolumn{2}{|c|}{ Puntuación } \\
\hline Aspectos del sujeto & $\underline{\text { SI (1) }}$ & $\underline{\mathrm{NO}(0)}$ \\
\hline 1.- ¿Está organizada la vigilancia de manera que se definen claramente: & & \\
\hline Fuentes. & - & - \\
\hline Niveles o escalones de organización (consolidación y análisis). & - & - \\
\hline Flujo de información. & - & \\
\hline Periodicidad del análisis. & - & - \\
\hline Retroalimentación. & — & - \\
\hline 2.- ¿Están definidos con claridad los objetivos? & & \\
\hline del nivel local. & - & - \\
\hline de otros niveles (municipal, provincial y nacional). & - & - \\
\hline 3.- ¿Se corresponde con la estructura y organización del Sistema de Salud? & - & - \\
\hline 4.- ¿Se ajusta su diseño a las necesidades de vigilancia del territorio? & - & - \\
\hline $\begin{array}{l}\text { 5.- ¿Cuenta el sistema con el personal necesario y suficiente para su adecuado desem- } \\
\text { peño? }\end{array}$ & 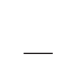 & - \\
\hline 6.- ¿Tienen los participantes en la vigilancia la formación y competencia adecuada? & - & - \\
\hline $\begin{array}{l}\text { 7.- ¿Están bien definidas y recogidas por escrito las funciones de cada participante y } \\
\text { componente del sistema? }\end{array}$ & - & - \\
\hline $\begin{array}{l}\text { 8.- ¿Se dispone de los equipos, instrumentos y materiales necesarios para el funciona- } \\
\text { miento del sistema (teléfono, correo, computadoras, modelos, etc.? }\end{array}$ & - & - \\
\hline Aspectos del objeto. & & \\
\hline 1.- ¿Está bien definida la población bajo vigilancia? & - & - \\
\hline $\begin{array}{l}\text { 2.- ¿Está delimitado el período de tiempo para el cual se recogerán y analizarán los da- } \\
\text { tos? }\end{array}$ & - & - \\
\hline $\begin{array}{l}\text { 3.- ¿Son conocidos las condiciones de la comunidad bajo vigilancia, en cuanto a sus ca- } \\
\text { racterísticas: }\end{array}$ & 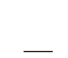 & - \\
\hline demográficas. & - & - \\
\hline socioeconómicas. & - & - \\
\hline Ambientales. & - & - \\
\hline $\begin{array}{l}\text { 4.- ¿Se han identificado los principales problemas de salud que afectan a esa comuni- } \\
\text { dad? }\end{array}$ & - & - \\
\hline
\end{tabular}




\begin{tabular}{|c|c|c|}
\hline Evaluación del proceso & \multicolumn{2}{|c|}{ Puntuación } \\
\hline Simplicidad. & $\underline{\text { SI (1) }}$ & $\underline{\mathrm{NO}(0)}$ \\
\hline 1.- ¿Son excesivas en cuanto a su cantidad o volumen: & & \\
\hline los datos a recoger. & - & - \\
\hline las fuentes de las que proceden dichos datos. & - & - \\
\hline el número de requisitos y documentos a confeccionar para el reporte. & - & - \\
\hline el tiempo global dedicado a las actividades del sistema? & - & - \\
\hline $\begin{array}{l}\text { 2.- ¿Resultan complejos o engorrosos los elementos y actividades de vigilancia en } \\
\text { cuanto a: }\end{array}$ & & \\
\hline tipo de datos. & - & - \\
\hline características de las fuentes. & - & - \\
\hline métodos para la transmisión de los datos. & - & - \\
\hline forma de consolidar y analizar los datos. & - & - \\
\hline forma de difundir y comunicar los resultados? & - & - \\
\hline $\begin{array}{l}\text { 3.- ¿Existen aspectos que complican y afectan la eficiencia del sistema, porque se ob- } \\
\text { serva: }\end{array}$ & & \\
\hline duplicidad de información. & - & - \\
\hline se recogen datos que no son analizados o utilizados. & - & - \\
\hline los distintos niveles y participantes recogen y analizan los datos sin intervenir? & - & - \\
\hline Aceptabilidad. & & \\
\hline $\begin{array}{l}\text { 1.- ¿Cumplen los participantes con las actividades por el sistema (recogida, transmi- } \\
\text { sión, análisis y comunicación)? }\end{array}$ & _ & - \\
\hline $\begin{array}{l}\text { 2.- ¿Las actividades realizadas tienen la calidad requerida para garantizar la eficiencia } \\
\text { del sistema: rapidez, regularidad, suficiencia de datos)? }\end{array}$ & 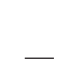 & - \\
\hline 3.- ¿Están satisfechos los usuarios por los resultados que le brinda el sistema? & - & - \\
\hline Flexibilidad. & & \\
\hline $\begin{array}{l}\text { 1.- ¿Permitió el sistema incorporar un nuevo grupo de datos para evaluar o vigilar un } \\
\text { nuevo problema? }\end{array}$ & 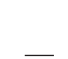 & 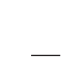 \\
\hline $\begin{array}{l}\text { 2.- ¿Implicó la introducción o modificación de algún elemento alteraciones en la orga- } \\
\text { nización del sistema? }\end{array}$ & 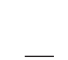 & - \\
\hline $\begin{array}{l}\text { 3.- ¿Resultó algún cambio en el proceso de vigilancia un gasto excesivo para el siste- } \\
\text { ma? }\end{array}$ & 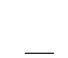 & - \\
\hline Integralidad & & \\
\hline $\begin{array}{l}\text { 1.- ¿Reconoce el sistema todos los factores que influyen en los problemas detectados o } \\
\text { sometidos a vigilancia como: biológicos, psicológicos, socioeconómicos, ambien- } \\
\text { tales y de atención médica? }\end{array}$ & - & - \\
\hline $\begin{array}{l}\text { 2.- ¿Están preparados los participantes en el sistema para actuar con el enfoque inte- } \\
\text { gral? }\end{array}$ & 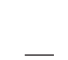 & - \\
\hline 3.- ¿Son abordados los eventos de salud en el individuo, la familia y la comunidad? & - & - \\
\hline
\end{tabular}




\begin{tabular}{|c|c|c|c|c|c|}
\hline$\underline{\text { Sensibilidad }}$ & $\frac{\text { Muy baja }}{(0)}$ & $\frac{\text { Media Baja }}{\text { (1) }}$ & $\frac{\text { Media }}{(2)}$ & $\frac{\text { Media alta }}{(3)}$ & $\frac{\text { Alta }}{(4)}$ \\
\hline 1.- índice de casos detectados/ocurridos & - & - & - & - & - \\
\hline 2.- Índice de casos detectados fuera del área/ocurridos & - & - & - & - & - \\
\hline Valor predictivo positivo. & $\frac{\text { Muy bajo }}{(0)}$ & $\frac{\text { Medio bajo }}{\text { (1) }}$ & $\frac{\text { Medio }}{(2)}$ & $\frac{\text { Medio alto }}{\text { (3) }}$ & $\frac{\text { Alto }}{(4)}$ \\
\hline $\begin{array}{l}\text { 1.- Relación de casos verdaderos positivos (confirma- } \\
\text { dos)/total de casos reportados como positivos (sospe- } \\
\text { chosos). }\end{array}$ & - & - & - & - & - \\
\hline Oportunidad. (Para el sistema Alerta-acción) * & $\frac{\begin{array}{c}\text { Muy } \\
\text { demorado }\end{array}}{(0)}$ & $\frac{\underline{\text { Medio }}}{\text { demorado }}$ & $\frac{\text { Demorado }}{(2)}$ & Ágil (3) & $\frac{\text { Muy ágil }}{\text { (4) }}$ \\
\hline $\begin{array}{l}\text { 1.- Rapidez con que se transmite y analiza la información } \\
\text { (etapas o pasos): }\end{array}$ & & & & & \\
\hline Primer paso. & - & - & - & - & - \\
\hline Segundo paso. & - & - & - & - & - \\
\hline Tercer paso. & - & - & - & - & - \\
\hline $\begin{array}{l}\text { 2.- ¿Se usan medios que agilizan el procesamiento de la } \\
\text { información: computación, correo electrónico, etc.? }\end{array}$ & - & - & - & - & \\
\hline Capacidad de autorrespuesta. & $\frac{\text { Nunca }}{(0)}$ & $\frac{\text { Casi nunca }}{(1)}$ & $\frac{\text { A veces }}{(2)}$ & $\frac{\text { Casi siempre }}{(3)}$ & $\frac{\text { Siempre }}{(4)}$ \\
\hline $\begin{array}{l}\text { 1.- ¿Se toman las medidas para actuar sobre los proble- } \\
\text { mas identificados? }\end{array}$ & - & - & - & - & - \\
\hline $\begin{array}{l}\text { 2.- ¿Se ejecutan plenamente las acciones establecidas por } \\
\text { la vigilancia? } \\
\text { 3.- ¿Se obtiene la intervención y apoyo intersectorial ne- } \\
\text { cesario? }\end{array}$ & - & - & - & - & - \\
\hline $\begin{array}{l}\text { 4.- ¿Participa la población en la ejecución de las medidas } \\
\text { orientadas que así lo requieren? } \\
\text { 5.- ¿Se logra controlar o eliminar el problema para el cual } \\
\text { se aplican las acciones recomendadas? }\end{array}$ & - & - & - & - & - \\
\hline Representatividad & $\frac{\text { Nunca }}{(0)}$ & $\frac{\text { Casi nunca }}{\text { (1) }}$ & $\frac{\text { A veces }}{(2)}$ & $\frac{\text { Casi siempre }}{(3)}$ & $\frac{\text { Siempre }}{(4)}$ \\
\hline $\begin{array}{l}\text { 1.- ¿Se corresponden los datos obtenidos con el compor- } \\
\text { tamiento de ese problema en la población bajo vigi- } \\
\text { lancia, en cuanto a: }\end{array}$ & & & & & \\
\hline tiempo. & - & - & - & - & - \\
\hline espacio. & - & - & - & - & - \\
\hline Persona según: edad & - & - & - & - & - \\
\hline Sexo & - & - & - & - & - \\
\hline grupo étnico & - & - & - & - & - \\
\hline grupo social & - & - & - & - & - \\
\hline grupo de riesgo o expuesto & - & _ & - & - & - \\
\hline
\end{tabular}




\begin{tabular}{|c|c|c|}
\hline Evaluación de los resultados & \multicolumn{2}{|c|}{ Puntuación } \\
\hline $\begin{array}{l}\text { ¿Puede el sistema: } \\
\text { 1. Detectar brotes o epidemias? } \\
\text { 2. Hacer predicciones o establecer la tendencia de determinado problema? } \\
\text { 3. Proporcionar la información necesaria sobre el comportamiento del problema bajo vigilancia: inci- } \\
\text { dencia, letalidad, mortalidad, riesgo, etc.? } \\
\text { 4. Identificar factores de riesgo asociados a una enfermedad o problema? } \\
\text { 5. Formular hipótesis causales? } \\
\text { 6. Estimular investigaciones epidemiológicas sobre algún problema para su control y prevención? } \\
\text { 7. Contribuir al perfeccionamiento de la práctica médica de todos los componentes y participantes? } \\
\text { 8. Detectar cambios en el comportamiento de agentes patógenos? } \\
\text { 9. Detectar eventos nuevos o desconocidos, no esperados anticipando situaciones emergentes? } \\
\text { 10. Evaluar los efectos de las medidas de control? } \\
\text { ¿El sistema resulta útil a otros usuarios (Gobierno, instituciones estatales: seguridad social, } \\
\text { de trabajo, economía, etc.)? } \\
\text { ¿Aporta información útil para la toma de decisiones? } \\
\text { ¿Utilizan los usuarios la información generada por el sistema? }\end{array}$ & $\underline{\text { SI (1) }}$ & $\underline{\mathrm{NO}(0)}$ \\
\hline
\end{tabular}


Anexo 2

Encuesta a participantes en el proceso de vigilancia

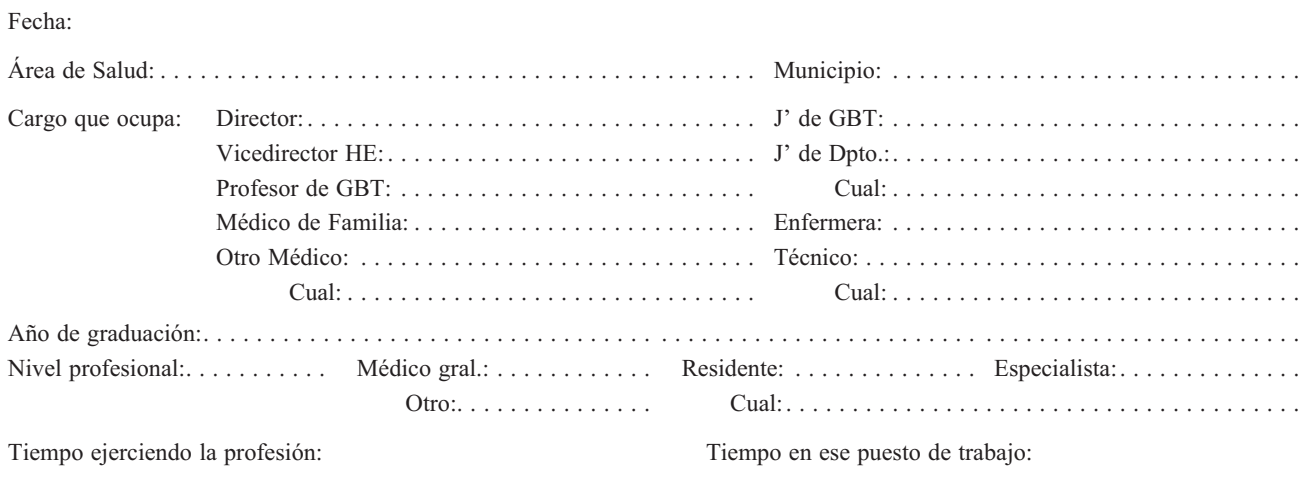

1.- ¿Ha participado en cursos de postgrado en especialidades de Higiene, Epidemiología O Salud Pública de más de 40 horas (Maestrías, Diplomados y cursos de tres meses o más), que incluyan capacitación o entrenamiento en vigilancia epidemiológica, en los últimos cuatro años?
$\mathrm{Si} \ldots .$.
No

Curso:

N.

2.- Considera que el reporte de los datos de notificación de enfermedades y otros daños es complicado (excesiva cantidad de datos, 1lenado innecesario de modelos, informes, etc.).

$\mathrm{Si}$

No

3.- Realiza usted el reporte de los problemas según lo establecido?
Siempre:
A veces:
Nunca:

4.- ¿Recibe alguna información periódica sobre la situación de salud en su área, del municipio u otros territorios?

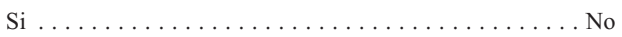

5.- ¿En qué forma recibe esta información?: Por escrito, Reporte o Boletín periódico: .

Oral, directamente de autoridades de salud:

En reuniones o encuentros para ese fin:.

6.- ¿Con qué frecuencia recibe esta información?

Diaria:

Semanal:

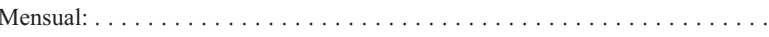

Trimestral:.

Otra: .

7.- ¿Podría ustes indicar cuales son los problemas de salud más relevantes en su Área?

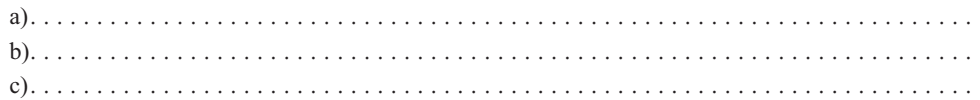

8.- En su opinión, ¿la vigilancia epidemiológica en su Área es útil?

$\mathrm{Si} \ldots \ldots \ldots \ldots \ldots \ldots \ldots \ldots \ldots \ldots \ldots$ 
Estimado compantero quisiéramos conocer sus opiniones sobre el proceso de difiasión de la información relacionada con los problemas de salud. Por favor conteste las preguntas sobre ed tema que a continuación le formularemos.

Fecha:

Area de Salud: Municipio:

Organizacion o sector que representa:

Cargo que ocupa:

Tiempo que lleva en esa responsabilidad:

1. Recibe alguna información sobre el comportamiento de los problemas relacionados con La situación de salud de su comunidad: Si No

\section{Si la respuesta es positiva.}

2. Cómo recibe esa información: Por escrito, Reporte o Boletin periódicot

Oral directamente de autoridades de salud:

En reuniones o encuentros parn ese fin:

Usted la busca activamente:

3. Cuales soo los problemss de salud más importantes en su territorio?

a.

b.

c.

4. ¿Que valor le atribuye a la información que recibe en su trabajo como representante comunitatio?
Muy úril
Util
Poco dril
No es ùtil

5. ¿ڤHa sido provechosa la información recibida para la toma de decisiones en relación con su labor cotnunitaria?

Siempre

A veces

Nunca 


\section{BIBLIOGRAFÍA}

1. Rodríguez Milord D. Sistema de Vigilancia en Salud. UATS, Area de Higiene y Epidemiología. La Habana: MINSAP; 1994.

2. Starfield B. Primary Care. Concept, evaluation and policy. Oxford University Press; 1992.

3. Donabedian A. La calidad de la atención médica. México: La Prensa Médica Mexicana;1984.

4. OPS/OMS. Evaluación de la tecnología empleada en la atención de la salud. Rev Panam Salud Publica 1997; 2(5):363-372.

5. Levy BS, Matine J, Washburn. Intensive hepatitis surveillance in Minnesota: methods and results. Am J Epidemiol 1977; 105:127-134.

6. Choi K, Thacker S. An Evaluation of influenza mortality surveillance 1962-1979. II Percentage of pneumonia and influenza deaths as an indicator of influenza activity. Am J Epidemiol 1981; 113:227-35.

7. Wharton M, Price W, Hoesly F, Woolard D, White K, Greene C, Mc Nabb S. Evaluation of a method for detecting outbreaks of diseases in six states. Am J Prev Med 1993; 9:45-9.

8. Kircher T, Nelson J, Burdo H. The autopsy as an meassure of accuracy of the deaths certificate. N Engl J Med 1985; 313:1263-9.

9. Coll Jorda D, Artegoitia Axpe JM, Martínez Navarro F. Evaluación de la vigilancia epidemiológica de la brucelosis en la comunidad autónoma del País Vasco. Rev Esp Salud Publica, 1999; 71(2):45-52.

10. Birbaun D. Recorded criteria as a «gold standard» for sensitivity and specificity estimates of surveillance of nosocomial infection: a novel method to measure job performance. Infect Control Hosp Epidemiol 1996; 17(6):345-6.

11. Gary HE, Sanders R, Pallansch MA. A theoretical framework for evaluating sensitivity of surveillance for detecting wild poliovirus: II. Factors affecting detection sensitivity in a population with circulating wild poliovirus. Infect Dis 1997 : 175 suppl 1:s141-5.
12. German RR. Sensitivity and predictive positive measurements for public health surveillance systems. Epidemiology 2000; 11(6):720-7.

13. Klauke DN, Beuhler JW, Thacker SB et al. Guidelines for Evaluations Surveillance Systems. US Department of Health and Human Services. MMWR 1988; 37 (supl-5):2.

14. Batista R. Propuesta metodológica para la evaluación de la vigilancia en la APS. [Tesis de Maestría en Epidemiología]. Instituto de Medicina Tropical Pedro Kourí. La Habana; 1996.

15. González Ochoa E. Diseño y evaluación de sistemas de vigilancia. En: Salud Publica. Colectivo de autores. La Habana: Editorial Ciencias Medicas; 2000.

16. Batista Moliner R, González Ochoa E. Evaluación de la vigilancia en la Atención Primaria de Salud: una propuesta metodológica. Rev Cubana Med Trop 2000; 52(1):55-65.

17. MINSAP. Programa de Prevención y control de la Tuberculosis. Servimpres La Habana: MINSAP; 1998.

18. MINSAP. Programa de Prevención y control de las Enfermedades de Transmisión Sexual. Servimpres. La Habana: MINSAP; 1998.

19. MINSAP. Programa de Prevención y control de la Leptospirosis Humana. Servimpres. La Habana: MINSAP; 1998.

0. Lóriga Bencomo A., Fariñas Reinoso AT., Acosta Rodríguez N. Evaluación rápida del sistema de vigilancia de la sífilis congénita en el municipio Pinar del Río, 1996. [Tesis para optar pos el título de Máster en Salud Pública] Universidad de la Habana; 1997.

21. Blanco Mederos Z., Fariñas Reinoso AT. Evaluación rápida del sistema de vigilancia de la sífilis congénita en el municipio San Cristóbal, 1996. [Tesis para optar por el título de especialista de Higiene y Epidemiología]. Universidad de la Habana; 1997.

22. Gómez de Haz, H; Fariñas Reinoso AT. Evaluación de la vigilancia de la sífilis congénita en Ciudad de La Habana, 1996. Rev Cub Salud Publica 1998; 24(2):73-7. 